\title{
On Sufficient Conditions for Chaotic Behavior of Multidimensional Discrete Time Dynamical System
}

\author{
Nor Syahmina Kamarudin ${ }^{1}[]^{-S y a h i d a ~ C h e ~ D z u l-K i f l i ~}{ }^{1}$
}

Received: 24 September 2020 / Revised: 21 January 2021 / Accepted: 22 March 2021 /

Published online: 3 April 2021

(c) The Author(s) 2021

\begin{abstract}
In this work, we look at the extension of classical discrete dynamical system to multidimensional discrete-time dynamical system by characterizing chaos notions on $\mathbb{Z}^{d}$-action. The $\mathbb{Z}^{d}$-action on a space $X$ has been defined in a very general manner, and therefore we introduce a $\mathbb{Z}^{d}$-action on $X$ which is induced by a continuous map, $f: \mathbb{Z} \times X \rightarrow X$ and denotes it as $T_{f}: \mathbb{Z}^{d} \times X \rightarrow X$. Basically, we wish to relate the behavior of origin discrete dynamical systems $(X, f)$ and its induced multidimensional discrete-time $\left(X, T_{f}\right)$. The chaotic behaviors that we emphasized are the transitivity and dense periodicity property. Analogues to these chaos notions, we consider $k$-type transitivity and $k$-type dense periodicity property in the multidimensional discrete-time dynamical system. In the process, we obtain some conditions on $\left(X, T_{f}\right)$ under which the chaotic behavior of $\left(X, T_{f}\right)$ is inherited from the original dynamical system $(X, f)$. The conditions varies whenever $f$ is open, totally transitive or mixing. Some examples are given to illustrate these conditions.
\end{abstract}

Keywords $\mathbb{Z}^{d}$-action $\cdot k$-Type transitive $\cdot$ Dense $k$-type periodic points $\cdot k$-Type Devaney chaos

Mathematics Subject Classification 37B99 $\cdot$ 54H20

Communicated by Rosihan M. Ali.

Syahida Che Dzul-Kifli

syahida@ukm.edu.my

Nor Syahmina Kamarudin

minasyahmina@gmail.com

1 Department of Mathematical Sciences, Faculty of Science and Technology, Universiti Kebangsaan Malaysia, 43600 UKM Bangi, Selangor, Malaysia 


\section{Introduction}

A $\mathbb{Z}^{d}$-action on a topological space $X$ is also known as a multidimensional discretetime dynamical system. The goal of this study on the dynamics of $\mathbb{Z}^{d}$-action is similar to the study on the classical theory of discrete dynamical system ( $\mathbb{Z}$-action, $\mathbb{N}$-action) which is to understand the nature of orbits in large iterations. It is necessary to understand the chaotic properties within the multidimensional discrete-time dynamical system, as for classical discrete dynamical system. However, the study on chaos theory in multidimensional case is far from complete and more chaos terms needed to be clearly defined in analogue to the classical chaos theory.

The existence of $k$-type chaos notion was firstly started by Oprocha who studied about chain recurrences in multidimensional discrete-time dynamical systems [5]. In order to work in $\mathbb{Z}^{d}$-action case, Oprocha had created a special inequality which will divide all limit points into $2^{d}$ different limit sets. They are called as $k$-type limit sets, where $k$ is said to be a number of the appropriate $d$-dimensional quarter. The ideas of $k$-type limit sets had given Shah and Das to introduce some other kinds of chaos notions.

In multidimensional chaos theory, Shah and Das introduced $k$-type Devaney chaos, $k$-type periodic points, $k$-type transitive, $k$-type sensitive dependence on initial condition, $k$-type mixing and many more [7-9]. The definitions are mostly analogue to the chaos notions (Devaney chaos, periodic points, transitivity etc.) which are considered in the classical dynamical system. We had known clearly that the sensitive dependence on initial condition is redundant to describe the Devaney chaos of a system of infinite metric space [1]. Then, the same goes to $\mathbb{Z}^{d}$-action on infinite metric space, Shah and Das has proved that $k$-type sensitive dependence on initial condition is also redundant for $k$-type Devaney chaos [8].

In another perspective, Shah and Das showed that conjugacy preserves $k$-type exact, $k$-type weak mixing and $k$-type Devaney chaos [8]. In addition, they found that the uniform conjugacy of $\mathbb{Z}^{d}$-actions preserves $k$-type densely chaos and $k$-type Auslander-Yorke chaos. In [9], they also proved that $k$-type collective sensitivity and $k$-type collective Auslander-Yorke chaotic are preserved under uniform conjugacy.

Besides, Shah and Das [7] explored the relationship between $\mathbb{Z}^{d}$-action on a space $X$ with its induced action on hyperspace $K(X)$ which is also known as a collection of compact subsets of $X$. It was found that there exist relationships between the two actions in terms of dense set of periodic points, dense set of $k$-type recurrent points, $k$-type transitive, $k$-type sensitive dependence on initial condition, $k$-type mixing and $k$-type weak mixing.

Note that $\mathbb{Z}$ and $\mathbb{Z}^{d}$ are the topological groups, and therefore there is a study on general action called a group action. The group action is defined in a similar way as $\mathbb{Z}^{d}$-action but topological group $G$ is used instead of $\mathbb{Z}^{d}$. Barzanouni et al. [2] showed that for an action of group, if it is expansive then the action of its syndetic subgroup is also expansive. Besides, they also proved that conjugacy between two group actions preserve the expansive property.

In $\mathbb{Z}^{d}$-action case, Shah had defined the concept of generator and studied on the expansive property of $\mathbb{Z}^{d}$-action [6]. The study proved a $\mathbb{Z}^{d}$-action is expansive if and 
only if it has a generator. Futher, the study had also prove several properties of an expansive $\mathbb{Z}^{d}$-action such as the characteristic of its expansive constants.

There is also an interest to $\mathbb{Z}^{d}$-action on product of two spaces. Shah and Das [8] found equivalent relation between $\mathbb{Z}^{d}$-action on product space with $\mathbb{Z}^{d}$-action on each space with $k$-type sensitive dependence on initial condition, $k$-type mixing and $k$-type transitive.

In a more particular view, Kim and Lee focused on $\mathbb{Z}^{2}$-action and introduced the notions of $k$-type limit sets and $k$-type nonwandering sets. They found that $k$-type nonwandering points of a $\mathbb{Z}^{2}$-action can be decomposed into a disjoint union of closed and $T$-invariant sets such that each of the sets is topologically $k$-type transitive if $T$ is expansive and has shadowing property [4].

The $\mathbb{Z}^{d}$-action on a space $X$ induced by a continuous map was introduced in [3] and we verified a relation of dynamics of $\mathbb{Z}^{d}$-action and transitive shift map. In 1-step shifts of finite type over two symbols, we proved that the $\mathbb{Z}^{d}$-action induced by the shift map is $k$-type transitive for all $k \in\left\{1,2, \ldots, 2^{d}\right\}$ whenever the shift map is topologically transitive or satisfies some sufficient conditions.

\section{Preliminary Definitions}

In this section, we discuss some definitions which will be used in this article. Let $(X, f)$ be a discrete dynamical system with a continuous map $f$ on $X$ into itself and the space $X$ that we consider is always a metric space.

A $\mathbb{Z}^{d}$-action on a space $X$ is a continuous map $T: \mathbb{Z}^{d} \times X \rightarrow X$ such that

(i) $T(\mathbf{0}, x)=x$, for all $x \in X$,

(ii) $T(\mathbf{n}, T(\mathbf{m}, x))=T(\mathbf{n}+\mathbf{m}, x)$, for all $\mathbf{n}, \mathbf{m} \in \mathbb{Z}^{d}$ and for all $x \in X$.

For a $\mathbb{Z}^{d}$-action, $T: \mathbb{Z}^{d} \times X \rightarrow X$, the map $T^{\mathbf{n}}: X \rightarrow X$ is defined by $T^{\mathbf{n}}(x)=$ $T(\mathbf{n}, x)$ for all $\mathbf{n} \in \mathbb{Z}^{d}$ and $x \in X$. Note that the map $T^{\mathbf{n}}$ is a homeomorphism on $X$ [7].

In classical topological dynamics study of $(X, f)$, we have that $f: X \rightarrow X$ is topologically transitive if for every pair of open sets $U$ and $V$ of $X$, there exists an integer $n>0$ such that $f^{n}(U) \cap V \neq \varnothing$. To define a correlated topologically transitive (and some other chaos notions) in multidimensional case, we have to specify an order in $\mathbb{Z}^{d}$. We let $k \in\left\{1,2,3, \ldots, 2^{d}\right\}$ and $k^{\prime} \in\{0,1\}^{d}$ such that $k=1+\sum_{i=1}^{d} k_{i}^{\prime} 2^{i-1}$. Let $\mathbf{x}=\left(x_{1}, x_{2}, \ldots, x_{d}\right) \in \mathbb{Z}^{d}$ and $\mathbf{y}=\left(y_{1}, y_{2}, \ldots, y_{d}\right) \in \mathbb{Z}^{d}$. We say that $\mathbf{x}>^{k} \mathbf{y}$ if $(-1)^{k_{i}^{\prime}} x_{i}>(-1)^{k_{i}^{\prime}} y_{i}$ for $i \in\{1,2, \ldots, d\}[5]$. Then, a $\mathbb{Z}^{d}$-action $T: \mathbb{Z}^{d} \times X \rightarrow X$ is said to be $k$-type transitive if for every open set $U$ and $V$ of $X$, there exists $\mathbf{n}>^{k} \mathbf{0}$ such that $T^{\mathbf{n}}(U) \cap V \neq \emptyset$ where $\mathbf{n} \in \mathbb{Z}^{d}[7]$.

For a map $f: X \rightarrow X$, a point $x$ is said to be periodic point of $f$ (with period $n$ ) if there exists an integer $n>0$ such that $f^{n}(x)=x$. Analogously, in multidimensional case,a point $x \in X$ is called a $k$-type periodic point if there is an $\mathbf{n}>^{k} \mathbf{0}$ satisfying $T^{\mathbf{n}}(x)=x$ where $\mathbf{n} \in \mathbb{Z}^{d}[7]$.

On an infinite metric space, a continuous map on it is said to be Devaney chaotic if it is transitive and has dense periodic points [1]. In multidimensional case, a $\mathbb{Z}^{d}$-action 
is said to be k-type Devaney chaotic if it is $k$-type transitive and has dense $k$-type periodic points [8].

The dynamical system $(X, f)$ is said to be totally transitive if for every $n \in \mathbb{N}$ and every pair of open sets $U$ and $V$ of $X$, there exists $k \in \mathbb{N}$ such that $\left(f^{n}\right)^{k}(U) \cap V \neq \emptyset$. The dynamical system $(X, f)$ is said to be topologically mixing if for any pair of nonempty open sets $U$ and $V$, there exists an $N>0$ such that $f^{n}(U) \cap V \neq \emptyset$ for all $n>N$. Note that a map which is totally transitive or topologically mixing is also topologically transitive.

This work is started by specifying $\mathbb{Z}^{d}$-action on a space $X$ which is induced by a continuous map $f$ on $X$ into itself. This new $\mathbb{Z}^{d}$-action is a generalization of examples of $\mathbb{Z}^{d}$-actions considered by Shah and Das [8] and others. The examples of $\mathbb{Z}^{d}$-action is defined with respect to a continuous map and another function $r^{\prime}(\mathbf{n})=n_{1}+n_{2}+\ldots+n_{d}$ for $\mathbf{n}=\left(n_{1}, n_{2}, \ldots, n_{d}\right)$. By generalising $r^{\prime}$ to $r(\mathbf{n})=h_{1} n_{1}+h_{2} n_{2}+\ldots+h_{d} n_{d}$ for some integers $h_{i}$, we then define a general form of $\mathbb{Z}^{d}$-action in Definition 1 . Therefore, it is worth to investigate the behavior of the $\mathbb{Z}^{d}$-action in dynamical sense.

Definition 1 Let $f: X \rightarrow X$ be a continuous map. $T_{f}: \mathbb{Z}^{d} \times X \rightarrow X$ is a map induced by $f$ and is defined by

$$
T_{f}^{\mathbf{n}}(x)=T_{f}(\mathbf{n}, x)=f^{r(\mathbf{n})}(x)
$$

for $\mathbf{n}=\left(n_{1}, n_{2}, \ldots, n_{d}\right) \in \mathbb{Z}^{d}, x \in X$ and $r: \mathbb{Z}^{d} \rightarrow \mathbb{Z}$ is a map of the form $r(\mathbf{n})=h_{1} n_{1}+h_{2} n_{2}+\ldots+h_{d} n_{d}$ where $h_{i} \in \mathbb{Z}$ for every $i \in\{1,2, \ldots, d\}$.

Remark $1 r: \mathbb{Z}^{d} \rightarrow \mathbb{Z}$ is a homomorphism if and only if $r(\mathbf{n})=h_{1} n_{1}+h_{2} n_{2}+\ldots+$ $h_{d} n_{d}$ for some integers $h_{i} \in \mathbb{Z}$ where $i \in\{1,2, \ldots, d\}$.

Lemma 1 The map, $T_{f}$ which is induced by continuous $f: X \rightarrow X$ and a homomorphism $r: \mathbb{Z}^{d} \rightarrow \mathbb{Z}$ is a $\mathbb{Z}^{d}$-action.

Proof Suppose that $r: \mathbb{Z}^{d} \rightarrow \mathbb{Z}$ is a homomorphism. Therefore, $r$ is given by $r\left(\left(n_{1}, n_{2}, \ldots, n_{d}\right)\right)=h_{1} n_{1}+h_{2} n_{2}+\ldots+h_{d} n_{d}$ for some $h_{i} \in \mathbb{Z}$ where $i \in\{1,2, \ldots, d\}$. Then,

$$
T_{f}\left(\left(0_{1}, 0_{2}, \ldots, 0_{d}\right), x\right)=f^{r\left(\left(0_{1}, 0_{2}, \ldots, 0_{d}\right)\right)}(x)=f^{0}(x)=x .
$$

Let $\mathbf{n}=\left(n_{1}, n_{2}, \ldots, n_{d}\right), \mathbf{m}=\left(m_{1}, m_{2}, \ldots, m_{d}\right) \in \mathbb{Z}^{d}$. Then, $T_{f}(\mathbf{n}$, $\left.T_{f}(\mathbf{m}, x)\right)=T_{f}\left(\mathbf{n}, f^{r(\mathbf{m})}(x)\right)=f^{r\left(\left(n_{1}, n_{2}, \ldots, n_{d}\right)\right)}\left(f^{r\left(\left(m_{1}, m_{2}, \ldots, m_{d}\right)\right)}(x)\right)$ $=f^{r\left(\left(n_{1}, n_{2}, \ldots, n_{d}\right)\right)+r\left(\left(m_{1}, m_{2}, \ldots, m_{d}\right)\right)}(x)=f^{r\left(\left(n_{1}+m_{1}, n_{2}+m_{2}, \ldots, n_{d}+m_{d}\right)\right)}(x)$ $=T_{f}\left(\left(n_{1}+m_{1}, n_{2}+m_{2}, \ldots, n_{d}+m_{d}\right), x\right)=T_{f}(\mathbf{n}+\mathbf{m}, x)$. Therefore, $T_{f}$ is a $\mathbb{Z}^{d}$-action.

Here are some examples of the $\mathbb{Z}^{d}$-action, $T_{f}$ which is induced by a continuous map $f$ on $X$.

Example 1 Shah and Das gave an example of map $T: \mathbb{Z}^{2} \times S^{1} \rightarrow S^{1}$ defined by $T\left(\left(n_{1}, n_{2}\right), \theta\right)=2^{n_{1}+n_{2}} \theta$ [8]. This map is a $\mathbb{Z}^{2}$-action induced by the doubling map $g: S^{1} \rightarrow S^{1}$ on the unit circle $S^{1}$ with $r\left(\left(n_{1}, n_{2}\right)\right)=n_{1}+n_{2}$. Therefore, $T=T_{g}$. It has been shown that $T$ is $k$-type transitive for $k \in\{1,2,3\}$ but not 4-type transitive. 
Kim and Lee also considered this example and shown that $\operatorname{Per}(T) \neq L^{k}(T)$ for $k \in\{1,2,3,4\}$ where $\operatorname{Per}(T)$ is the set of all periodic points and $L^{k}(T)$ is $k$-type limit set of $T$ [4].

Example 2 Define a map $f:[0,1] \rightarrow[0,1]$ by $f(x)=x-\frac{1}{10} \sin 2 \pi x$. Kim and Lee considered a $\mathbb{Z}^{2}$-action $T: \mathbb{Z}^{2} \times[0,1] \rightarrow[0,1]$ which be defined by $T_{f}\left(\left(n_{1}, n_{2}\right), x\right)=$ $f^{n_{1}+n_{2}}(x)$ [4]. Then, $T=T_{f}$ is a $\mathbb{Z}^{2}$-action induced by the map $f$ on the unit interval $[0,1]$ with $r\left(\left(n_{1}, n_{2}\right)\right)=n_{1}+n_{2}$. It has been shown that $L^{k}(T) \neq \Omega^{k}(T)$ for $k \in\{1,2,3,4\}$ where $\Omega^{k}(T)$ is the set of $k$-type nonwandering points of $T$.

Example 3 Let $\mathcal{A}=\{0,1\}$ and $\mathcal{A}^{\mathbb{Z}^{2}}$ be the set of all sequences $\mathbf{s}=\left\{s_{i}\right\}_{i \in \mathbb{N}}$ where $s_{i}$ is either 0 or 1 . Equipped with this space is a map called the shift map, $\sigma$ which shifts each sequences $\mathbf{S}=s_{0} s_{1} s_{2} \ldots$, one step to the left, i.e., $\sigma(\mathbf{s})=s_{1} s_{2} s_{3} \ldots$. Shah and Das defined the $\mathbb{Z}^{2}$-action, $\Sigma$ on $\mathcal{A}^{\mathbb{Z}^{2}}$ as follows: $\left(\Sigma^{\left(n_{1}, n_{2}\right)}(x)\right)=x_{n_{1}+n_{2}}$ [8]. Therefore, the $\mathbb{Z}^{2}$-action, $\Sigma$ is induced by the shift map $\sigma$ with $r\left(\left(n_{1}, n_{2}\right)\right)=n_{1}+n_{2}$. It has been shown that $\left(\mathcal{A}^{\mathbb{Z}^{2}}, \Sigma\right)$ is 1, 2, 3, 4-type Devaney chaotic.

It is natural to ask the relation between chaotic behavior of the dynamical system of $(X, f)$ and $\left(X, T_{f}\right)$. Therefore, we explore the relationship between the dynamics of these dynamical systems.

\section{Transitivity and $k$-type Transitivity}

According to Shah and Das, the $\mathbb{Z}^{2}$-action which induced by the doubling map on the unit circle $S^{1}, T_{g}$ as in Example 1 is $k$-type transitive for $k \in\{1,2,3\}$ but not 4-type transitive [8]. Since the doubling map is transitive, it then shows that the transitivity of $f$ on $X$ does not guarantee that $T_{f}$ is $k$-type transitive for all $k \in\left\{1,2, \ldots, 2^{d}\right\}$. Therefore, in this section, we derive some conditions on homomorphism $r$ so that $f$ passes this chaos notion to $T_{f}$.

Theorem 1 Let $f: X \rightarrow X$ be a transitive continuous map and $T_{f}: \mathbb{Z}^{d} \times X \rightarrow X$ be a $\mathbb{Z}^{d}$-action on $X$ induced by $f$. Suppose $k \in\left\{1,2, \ldots, 2^{d}\right\}$. If there exists $\boldsymbol{m}>^{k} \boldsymbol{O}$ such that $r(\boldsymbol{m})=1$, then $T_{f}$ is $k$-type transitive.

Proof Suppose that $f$ is transitive. Suppose that $\mathbf{m}>^{k} \mathbf{0}$ such that $r(\mathbf{m})=1$ where $k \in\left\{1,2, \ldots, 2^{d}\right\}$. Then, $r(\mathbf{m})=h_{1} m_{1}+h_{2} m_{2}+\ldots+h_{d} m_{d}=1$ where $h_{i} \in \mathbb{Z}$ for all $i \in\{1,2, \ldots, d\}$ and $\mathbf{m}=\left(m_{1}, m_{2}, \ldots, m_{d}\right) \in \mathbb{Z}^{d}$. Then, for all $n \in \mathbb{N}$, $n=n(1)=n\left(h_{1} m_{1}+h_{2} m_{2}+\ldots+h_{d} m_{d}\right)=h_{1}\left(n m_{1}\right)+h_{2}\left(n m_{2}\right)+\ldots+h_{d}\left(n m_{d}\right)$. Let $U$ and $V$ be nonempty open sets of $X$. Since $f$ is transitive, then there exists $l>0$ such that $f^{l}(U) \cap V \neq \emptyset$. Since $l>0$, then $l=h_{1}\left(\operatorname{lm}_{1}\right)+h_{2}\left(l m_{2}\right)+\ldots+h_{d}\left(l m_{d}\right)$. Choose $\mathbf{s}=\left(l m_{1}, l m_{2}, \ldots, l m_{d}\right)$. Since $\left(m_{1}, m_{2}, \ldots, m_{d}\right)>^{k} \mathbf{0}$, then $\mathbf{s}>^{k} \mathbf{0}$. Then, $T_{f}^{\mathbf{s}}(U) \cap V=f^{r(\mathbf{s})}(U) \cap V=f^{h_{1}\left(l_{1}\right)+h_{2}\left(l m_{2}\right)+\ldots+h_{d}\left(l_{d}\right)}(U) \cap V=f^{l}(U) \cap V \neq \emptyset$. Therefore, $T_{f}$ is $k$-type transitive.

To illustrate this result, let us consider $\mathbb{Z}^{2}$-actions on $X$ which is induced by $f$ and with four different cases of homomorphism $r\left(\left(n_{1}, n_{2}\right)\right)=a n_{1}+b n_{2}$, i.e., $r_{1}\left(\left(n_{1}, n_{2}\right)\right)=n_{1}+n_{2}, r_{2}\left(\left(n_{1}, n_{2}\right)\right)=n_{1}-n_{2}, r_{3}\left(\left(n_{1}, n_{2}\right)\right)=-2 n_{1}+n_{2}$ and $r_{4}\left(\left(n_{1}, n_{2}\right)\right)=-5 n_{1}-3 n_{2}$. The examples are explained in the following. 
Example 4 Let $f: X \rightarrow X$ be a continuous map and $T_{f}: \mathbb{Z}^{2} \times X \rightarrow X$ is defined by $T_{f}^{\mathbf{n}}(x)=T_{f}(\mathbf{n}, x)=f^{n_{1}+n_{2}}(x)$. If $f$ is transitive, then $T_{f}$ is 2, 3-type transitive but not necessarily 4-type transitive.

Proof If $k=2$, then we have $(-1,2)>^{2} \mathbf{0}$ such that $(-1)+2=1$. By Theorem $1, T_{f}$ is 2-type transitive whenever $f$ is transitive. If $k=3$, we have $(2,-1)>^{3} \mathbf{0}$ such that $2+(-1)=1$. Similarly, $T_{f}$ is 3-type transitive whenever $f$ is transitive. For $k=4$, consider the doubling map, $g$ on the unit circle $S^{1}$ as counterexample. By taking the doubling map $g$ as $g(\theta)=2 \theta$, then the $\mathbb{Z}^{2}$-action induced by $g$ is given by $T_{g}\left(\left(n_{1}, n_{2}\right), \theta\right)=2^{n_{1}+n_{2}} \theta$ whenever $r(\mathbf{n})=n_{1}+n_{2}$. Let $\alpha \in S^{1}$ such that $0 \leq \alpha \leq \frac{\pi}{2}$ (in radian) and $\left(m_{1}, m_{2}\right)>^{4} \mathbf{0}$. Then, $m_{1}+m_{2}<0$. Then, $T_{g}\left(\left(m_{1}, m_{2}\right), \alpha\right)=$ $2^{m_{1}+m_{2}} \alpha=2^{j} \alpha$ such that $j<0$. Then, $T_{g}\left(\left(m_{1}, m_{2}\right), \alpha\right)=\frac{1}{2^{-j}} \alpha \in\left[0, \frac{\pi}{2}\right]$. Thus, for any arc $U$ and $V$ in the first and third quadrant, respectively, $T_{g}^{\mathbf{m}}(U) \cap V=\emptyset$ for all $\mathbf{m}=\left(m_{1}, m_{2}\right)>^{4} \mathbf{0}$. Therefore, $T_{g}$ is not 4-type transitive.

Note that the occurrence of 1-type transitivity property is unknown.

Example 5 Let $f: X \rightarrow X$ be a continuous map and $T_{f}: \mathbb{Z}^{2} \times X \rightarrow X$ is defined by $T_{f}^{\mathbf{n}}(x)=T_{f}(\mathbf{n}, x)=f^{n_{1}-n_{2}}(x)$. If $f$ is transitive, then $T_{f}$ is 1, 4-type transitive but not necessarily 2 -type transitive.

Proof If $k=1$, then we have $(2,1)>^{1} \mathbf{0}$ such that $2-1=1$. By Theorem $1, T_{f}$ is 1-type transitive whenever $f$ is transitive. If $k=4$, we have $(-1,-2)>^{4} \mathbf{0}$ such that $(-1)-(-2)=1$. Similarly, $T_{f}$ is 4-type transitive whenever $f$ is transitive. For $k=2$, consider the doubling map, $g$ on the unit circle $S^{1}$ as counterexample. By taking the doubling map $g$ as $g(\theta)=2 \theta$, then the $\mathbb{Z}^{2}$-action induced by $g$ is given by $T_{g}\left(\left(n_{1}, n_{2}\right), \theta\right)=2^{n_{1}-n_{2}} \theta$ whenever $r(\mathbf{n})=n_{1}-n_{2}$. Let $\alpha \in S^{1}$ such that $0 \leq \alpha \leq \frac{\pi}{2}$ (in radian) and $\left(m_{1}, m_{2}\right)>^{2} \mathbf{0}$. Then, $m_{1}-m_{2}<0$. Then, $T_{g}\left(\left(m_{1}, m_{2}\right), \alpha\right)=$ $2^{m_{1}-m_{2}} \alpha=2^{j} \alpha$ such that $j<0$. Then, $T_{g}\left(\left(m_{1}, m_{2}\right), \alpha\right)=\frac{1}{2^{-j}} \alpha \in\left[0, \frac{\pi}{2}\right]$. Thus, for any arc $U$ and $V$ in the first and third quadrant respectively, $T_{g}^{\mathbf{m}}(U) \cap V=\emptyset$ for all $\mathbf{m}=\left(m_{1}, m_{2}\right)>^{2} \mathbf{0}$. Therefore, $T_{g}$ is not 2-type transitive.

Note that the occurrence of 3-type transitivity property is unknown.

Example 6 Let $f: X \rightarrow X$ be a continuous map and $T_{f}: \mathbb{Z}^{2} \times X \rightarrow X$ is defined by $T_{f}^{\mathbf{n}}(x)=T_{f}(\mathbf{n}, x)=f^{-2 n_{1}+n_{2}}(x)$. If $f$ is transitive, then $T_{f}$ is 1, 4-type transitive but not necessarily 3-type transitive.

Proof If $k=1$, we have $(1,3)>>^{1} \mathbf{0}$ such that $-2(1)+3=1$. By Theorem $1, T_{f}$ is 1-type transitive whenever $f$ is transitive. If $k=4$, we have $(-1,-1)>^{4} \mathbf{0}$ such that $-2(-1)+(-1)=1$. Similarly, $T_{f}$ is 4-type transitive whenever $f$ is transitive. For $k=3$, consider the doubling map, $g$ on the unit circle $S^{1}$ as counterexample. By taking the doubling map $g$ as $g(\theta)=2 \theta$, then the $\mathbb{Z}^{2}$-action induced by $g$ is given by $T_{g}\left(\left(n_{1}, n_{2}\right), \theta\right)=2^{-2 n_{1}+n_{2}} \theta$ whenever $r(\mathbf{n})=-2 n_{1}+n_{2}$. Let $\alpha \in S^{1}$ such that $0 \leq \alpha \leq \frac{\pi}{2}$ (in radian) and $\left(m_{1}, m_{2}\right)>^{3} \mathbf{0}$. Then, $-2 m_{1}+m_{2}<0$. Then, $T_{g}\left(\left(m_{1}, m_{2}\right), \alpha\right)=2^{-2 m_{1}+m_{2}} \alpha=2^{j} \alpha$ such that $j<0$. Then, $T_{g}\left(\left(m_{1}, m_{2}\right), \alpha\right)=$ $\frac{1}{2^{-j}} \alpha \in\left[0, \frac{\pi}{2}\right]$. Thus, for any arc $U$ and $V$ in the first and third quadrant respectively, $T_{g}^{\mathbf{m}}(U) \cap V=\emptyset$ for all $\mathbf{m}=\left(m_{1}, m_{2}\right)>^{3} \mathbf{0}$. Therefore, $T_{g}$ is not 3-type transitive. 
Note that the occurrence of 2-type transitivity property is unknown.

Example 7 Let $f: X \rightarrow X$ be a continuous map and $T_{f}: \mathbb{Z}^{2} \times X \rightarrow X$ is defined by $T_{f}^{\mathbf{n}}(x)=T_{f}(\mathbf{n}, x)=f^{-5 n_{1}-3 n_{2}}(x)$. If $f$ is transitive, then $T_{f}$ is 2, 3-type transitive but not necessarily 1-type transitive.

Proof If $k=2$, we have $(-2,3)>^{2} \mathbf{0}$ such that $-5(-2)-3(3)=1$. By Theorem 1 , $T_{f}$ is 2-type transitive whenever $f$ is transitive. If $k=3$, we have $(4,-7)>^{3} \mathbf{0}$ such that $-5(4)-3(-7)=1$. Similarly, $T_{f}$ is 3-type transitive whenever $f$ is transitive. For $k=1$, consider the doubling map, $g$ on the unit circle $S^{1}$ as counterexample. By taking the doubling map $g$ as $g(\theta)=2 \theta$, then the $\mathbb{Z}^{2}$-action induced by $g$ is given by $T_{g}\left(\left(n_{1}, n_{2}\right), \theta\right)=2^{-5 n_{1}-3 n_{2}} \theta$ whenever $r(\mathbf{n})=-5 n_{1}-3 n_{2}$. Let $\alpha \in S^{1}$ such that $0 \leq \alpha \leq \frac{\pi}{2}$ (in radian) and $\left(m_{1}, m_{2}\right)>^{1} \mathbf{0}$. Then, $-5 m_{1}-3 m_{2}<0$. Then, $T_{g}\left(\left(m_{1}, m_{2}\right), \alpha\right)=2^{-5 m_{1}-3 m_{2}} \alpha=2^{j} \alpha$ such that $j<0$. Then, $T_{g}\left(\left(m_{1}, m_{2}\right), \alpha\right)=$ $\frac{1}{2^{-j}} \alpha \in\left[0, \frac{\pi}{2}\right]$. Thus, for any arc $U$ and $V$ in the first and third quadrant respectively, $T_{g}^{\mathbf{m}}(U) \cap V=\emptyset$ for all $\mathbf{m}=\left(m_{1}, m_{2}\right)>^{1} \mathbf{0}$. Therefore, $T_{g}$ is not 1-type transitive.

Note that the occurrence of 4-type transitivity property is unknown. Gaps in the above examples are going to be clarified by using the next results. We obtain a sharper result for an open map and the condition found earlier is adjusted so that $T_{f}$ is $k$-type transitive (for all $k$ ) whenever $f$ is transitive.

Theorem 2 Let $f: X \rightarrow X$ be a transitive continuous open map and $T_{f}: \mathbb{Z}^{d} \times X \rightarrow$ $X$ be $a \mathbb{Z}^{d}$-action on $X$ induced by $f$. Suppose $k \in\left\{1,2, \ldots, 2^{d}\right\}$. If there exists $m \in \mathbb{N}$ such that for all integers $s \geq m$, there exists $\boldsymbol{n}^{\prime}>^{k} \boldsymbol{0}$ such that $r\left(\boldsymbol{n}^{\prime}\right)=s$ for $\boldsymbol{n}^{\prime} \in \mathbb{Z}^{d}$, then $T_{f}$ is $k$-type transitive.

Proof Let $m \in \mathbb{N}$ such that for all integers $s \geq m$, there exists $\mathbf{n}^{\prime}>^{k} \mathbf{0}$ such that $r\left(\mathbf{n}^{\prime}\right)=s$ where $\mathbf{n}^{\prime} \in \mathbb{Z}^{d}$ and $k \in\left\{1,2, \ldots, 2^{\bar{d}}\right\}$. Let $U$ and $V$ be nonempty open sets of $X$. Since $f$ is transitive, then there exists $l>0$ such that $f^{l}(U) \cap V \neq \varnothing$. Consider two cases. The first case is if $l \geq m$. Then, there exists $\mathbf{n}_{l}>^{k} \mathbf{0}$ such that $r\left(\mathbf{n}_{l}\right)=l$ where $\mathbf{n}_{l} \in \mathbb{Z}^{d}$. By taking $\mathbf{n}_{l}$, we will have $T_{f}^{\mathbf{n}_{l}}(U) \cap V=T_{f}\left(\mathbf{n}_{l}, U\right) \cap V=$ $f^{r\left(\mathbf{n}_{l}\right)}(U) \cap V=f^{l}(U) \cap V \neq \varnothing$. While the second case is if $l<m$. Since $f$ is an open map, then $f^{l}(U)$ is an open set. Since $f$ is transitive, then there exists $t \in \mathbb{N}$ such that $f^{l+t}(U) \cap V \neq \emptyset$. Then, we will eventually find $f^{p}(U) \cap V \neq \emptyset$ where $p \geq m$ and $p \geq l+t$. So, it will also be in the first case. By these two cases, we have completed the proof.

This result is then being used to fill up the gaps in the previous examples. To illustrate this, we consider similar examples of $r_{1}, r_{2}, r_{3}$ and $r_{4}$ in Examples 4-7.

Example 8 Let $f: X \rightarrow X$ be a continuous and open map. Let $T_{f}: \mathbb{Z}^{2} \times X \rightarrow X$ be defined by $T_{f}^{\mathbf{n}}(x)=T_{f}(\mathbf{n}, x)=f^{n_{1}+n_{2}}(x)$. If $f$ is transitive, then $T_{f}$ is 1, 2, 3-type transitive but not necessarily 4-type transitive.

Proof By Example 4, $T_{f}$ is 2, 3-type transitive but not necessarily 4-type transitive. For $k=1$, there is no $\left(n_{1}, n_{2}\right)>^{1} \mathbf{0}$ such that $n_{1}+n_{2}=1$. However, for all $l \geq 2$, we have $(l-1,1)>^{1} \mathbf{0}$ such that $(l-1)+1=l$. By Theorem 2 , if $f$ is a continuous and open map, then $T_{f}$ is 1-type transitive whenever $f$ is transitive. 
Example 9 Let $f: X \rightarrow X$ be a continuous and open map. Let $T_{f}: \mathbb{Z}^{2} \times X \rightarrow X$ be defined by $T_{f}^{\mathbf{n}}(x)=T_{f}(\mathbf{n}, x)=f^{n_{1}-n_{2}}(x)$. If $f$ is transitive, then $T_{f}$ is 1,3 , 4-type transitive but not necessarily 2-type transitive.

Proof By Example 5, $T_{f}$ is 1, 4-type transitive but not necessarily 2-type transitive. For $k=3$, there is no $\left(n_{1}, n_{2}\right)>^{3} \mathbf{0}$ such that $n_{1}-n_{2}=1$. However, for all $l \geq 2$, we have $(1,-(l-1))>^{3} \mathbf{0}$ such that $1-(-(l-1))=1-(-l+1)=l$. By Theorem 2 , if $f$ is a continuous and open map, then $T_{f}$ is 3-type transitive whenever $f$ is transitive.

Example 10 Let $f: X \rightarrow X$ be a continuous and open map. Let $T_{f}: \mathbb{Z}^{2} \times X \rightarrow X$ be defined by $T_{f}^{\mathbf{n}}(x)=T_{f}(\mathbf{n}, x)=f^{-2 n_{1}+n_{2}}(x)$. If $f$ is transitive, then $T_{f}$ is 1, 2, 4-type transitive but not necessarily 3-type transitive.

Proof By Example 6, $T_{f}$ is 1, 4-type transitive but not necessarily 3-type transitive. For $k=2$, there is no $\left(n_{1}, n_{2}\right)>^{2} \mathbf{0}$ such that $-2 n_{1}+n_{2}=1$. However, for all $l \geq 3$, we have $(-1, l-2)>^{2} \mathbf{0}$ such that $-2(-1)+(l-2)=l$. By Theorem 2 , if $f$ is a continuous and open map, then $T_{f}$ is 2-type transitive whenever $f$ is transitive.

Example 11 Let $f: X \rightarrow X$ be a continuous and open map. Let $T_{f}: \mathbb{Z}^{2} \times X \rightarrow X$ be defined by $T_{f}^{\mathbf{n}}(x)=T_{f}(\mathbf{n}, x)=f^{-5 n_{1}-3 n_{2}}(x)$. If $f$ is transitive, then $T_{f}$ is $2,3,4-$ type transitive but not necessarily 1-type transitive.

Proof By Example 7, $T_{f}$ is 2, 3-type transitive but not necessarily 1-type transitive. For $k=4$, there is no $\left(n_{1}, n_{2}\right)>^{4} \mathbf{0}$ such that $-5 n_{1}-3 n_{2}=1$. However, for all $l \geq 16$, we have three cases.

Case 1: If $l=10+3 j$ where $j \geq 2$, we have $\left(-2,-\frac{l-10}{3}\right)>^{4} \mathbf{0}$ such that $-5(-2)-$ $3\left(-\frac{l-10}{3}\right)=10+3\left(\frac{10+3 j-10}{3}\right)=10+3 j=l$.

Case 2: If $l=5+3 j$ where $j \geq 4$, we have $\left(-1,-\frac{l-5}{3}\right)>{ }^{4} \mathbf{0}$ such that $-5(-1)-$ $3\left(-\frac{l-5}{3}\right)=5+3\left(\frac{5+3 j-5}{3}\right)=5+3 j=l$.

Case 3: If $l=15+3 j$ where $j \geq 1$, we have $\left(-3,-\frac{l-15}{3}\right)>{ }^{4} \mathbf{0}$ such that $-5(-3)-$ $3\left(-\frac{l-15}{3}\right)=15+3\left(\frac{15+3 j-15}{3}\right)=15+3 j=l$.

By these three cases, we can say that for all $l \geq 16$, there exists $\mathbf{S}>^{4} \mathbf{0}$ such that $r(\mathbf{s})=l$. By Theorem 2, if $f$ is a continuous and open map, then $T_{f}$ is 4-type transitive whenever $f$ is transitive.

The implication in Theorem 2 is also true for totally transitive and topologically mixing map, as given by follows.

Theorem 3 Let $f: X \rightarrow X$ be a continuous totally transitive map and $T_{f}: \mathbb{Z}^{d} \times X \rightarrow$ $X$ be $a \mathbb{Z}^{d}$-action on $X$ induced by $f$. Suppose $k \in\left\{1,2, \ldots, 2^{d}\right\}$. If there exists $m \in \mathbb{N}$ such that for all integers $s \geq m$, there exists $\boldsymbol{n}^{\prime}>^{k} 0$ such that $r\left(\boldsymbol{n}^{\prime}\right)=s$ where $\boldsymbol{n}^{\prime} \in \mathbb{Z}^{d}$, then $T_{f}$ is $k$-type transitive.

Proof Let $m \in \mathbb{N}$ such that for all integers $s \geq m$, there exists $\mathbf{n}^{\prime}>^{k} \mathbf{0}$ such that $r\left(\mathbf{n}^{\prime}\right)=s$ where $\mathbf{n}^{\prime} \in \mathbb{Z}^{d}$ and $k \in\left\{1,2, \ldots, 2^{\bar{d}}\right\}$. Let $U$ and $V$ be nonempty open sets of $X$. Since $f$ is totally transitive, then $f^{n}$ is transitive for all $n \geq 1$. Choose 
$l=m$. Then, $f^{l}$ is transitive so there exists $j>0$ such that $f^{l+j}(U) \cap V \neq \emptyset$. Since $l+j \geq m$, then there exists $\mathbf{n}_{l}>^{k} \mathbf{0}$ such that $r\left(\mathbf{n}_{l}\right)=l+j$. By taking $\mathbf{n}_{l}$, we may have $T_{f}^{\mathbf{n}_{l}}(U) \cap V=T_{f}\left(\mathbf{n}_{l}, U\right) \cap V=f^{r\left(\mathbf{n}_{l}\right)}(U) \cap V=f^{l+j}(U) \cap V \neq \emptyset$. Therefore, $T_{f}$ is $k$-type transitive.

Corollary 1 Let $f: X \rightarrow X$ be a continuous topologically mixing map and $T_{f}$ : $\mathbb{Z}^{d} \times X \rightarrow X$ be a $\mathbb{Z}^{d}$-action on $X$ induced by $f$. Suppose $k \in\left\{1,2, \ldots, 2^{d}\right\}$. If there exists $m \in \mathbb{N}$ such that for all integers $s \geq m$, there exists $\boldsymbol{n}^{\prime}>^{k} 0$ such that $r\left(\boldsymbol{n}^{\prime}\right)=s$ where $\boldsymbol{n}^{\prime} \in \mathbb{Z}^{d}$, then $T_{f}$ is $k$-type transitive.

Proof Let $m \in \mathbb{N}$ such that for all integers $s \geq m$, there exists $\mathbf{n}^{\prime}>^{k} \mathbf{0}$ such that $r\left(\mathbf{n}^{\prime}\right)=s$ where $\mathbf{n}^{\prime} \in \mathbb{Z}^{d}$ and $k \in\left\{1,2, \ldots, 2^{d}\right\}$. Let $U$ and $V$ be nonempty open sets of $X$. Since $f$ is topologically mixing, then there exists $m^{\prime}>0$ such that $f^{e}(U) \cap V \neq \varnothing$ for all $e \geq m^{\prime}$. Choose integer $p$ which $p \geq m$ and $p \geq m^{\prime}$. Then, $f^{p}(U) \cap V \neq \emptyset$. Since $p \geq m$, then there exists $\mathbf{n}_{p}>^{k} \mathbf{0}$ such that $r\left(\mathbf{n}_{p}\right)=p$. By taking $\mathbf{n}_{p}$, we will have $T_{f}^{\mathbf{n}_{p}}(U) \cap V=T_{f}\left(\mathbf{n}_{p}, U\right) \cap V=f^{r\left(\mathbf{n}_{p}\right)}(U) \cap V=f^{p}(U) \cap V \neq \emptyset$. Therefore, $T_{f}$ is $k$-type transitive.

We use similar examples to illustrate these results and we omit the proofs as they are similar to the proofs in previous examples.

Example 12 Let $f$ be either totally transitive or topologically mixing and $T_{f}$ be a $\mathbb{Z}^{2}$-action on $X$ induced by $f$. Then, we have the following:

1. If $r\left(\left(n_{1}, n_{2}\right)\right)=n_{1}+n_{2}$, then $T_{f}$ is 1, 2, 3-type transitive but not necessarily 4-type transitive.

2. If $r\left(\left(n_{1}, n_{2}\right)\right)=n_{1}-n_{2}$, then $T_{f}$ is 1, 3, 4-type transitive but not necessarily 2-type transitive.

3. If $r\left(\left(n_{1}, n_{2}\right)\right)=-2 n_{1}+n_{2}$, then $T_{f}$ is 1,2, 4-type transitive but not necessarily 3-type transitive.

4. If $r\left(\left(n_{1}, n_{2}\right)\right)=-5 n_{1}-3 n_{2}$, then $T_{f}$ is 2, 3, 4-type transitive but not necessarily 1-type transitive.

\section{Dense Periodic Points and Dense $k$-type Periodic Points}

In this section, we show that the same conditions are used to guarantee that density property of periodic points and Devaney chaotic is inherited from $f$ to $T_{f}$.

Theorem 4 Let $f: X \rightarrow X$ be a continuous map with dense periodic points and $T_{f}: \mathbb{Z}^{d} \times X \rightarrow X$ be a $\mathbb{Z}^{d}$-action on $X$ induced by $f$. Suppose $k \in\left\{1,2, \ldots, 2^{d}\right\}$. If there exists $m \in \mathbb{N}$ such that for all integers $s \geq m$, there exists $\boldsymbol{n}^{\prime}>^{k} \boldsymbol{O}$ such that $r\left(\boldsymbol{n}^{\prime}\right)=s$ where $\boldsymbol{n}^{\prime} \in \mathbb{Z}^{d}$, then $T_{f}$ has dense $k$-type periodic points.

Proof Let $m \in \mathbb{N}$ such that for all integers $s \geq m$, there exists $\mathbf{n}^{\prime}>^{k} \mathbf{0}$ such that $r\left(\mathbf{n}^{\prime}\right)=s$ where $\mathbf{n}^{\prime} \in \mathbb{Z}^{d}$ and $k \in\left\{1,2, \ldots, \overline{2}^{d}\right\}$. Let $U$ be a nonempty open set of $X$. Since $(X, f)$ has dense periodic points, then there exists $y \in U$ such that $f^{l}(y)=y$ for some $l \in \mathbb{N}$. Consider two cases. The first case is if $l \geq m$, then 
there exists $\mathbf{n}_{l}>^{k} \mathbf{0}$ such that $r\left(\mathbf{n}_{l}\right)=l$ where $\mathbf{n}_{l} \in \mathbb{Z}^{d}$. By taking $\mathbf{n}_{l}$, we will have $T_{f}^{\mathbf{n}_{l}}(y)=T_{f}\left(\mathbf{n}_{l}, y\right)=f^{r\left(\mathbf{n}_{l}\right)}(y)=f^{l}(y)=y$. While the second case is if $l<m$, since $f^{l}(y)=y$, then $f^{t l}(y)=y$ for all $t \in \mathbb{N}$. Take $p l \geq m$ where $p \in \mathbb{N}$. Then, it will also be in the first case. By these two cases, we have completed the proof. Hence, $T_{f}$ has dense $k$-type periodic points.

It turns out that some type of the density of periodic points is guaranteed for the same cases considered in the previous section.

Example 13 Let $f$ has dense periodic points in $X$ and $T_{f}$ be a $\mathbb{Z}^{2}$-action on $X$ induced by $f$. Then, we have the following:

1. If $r\left(\left(n_{1}, n_{2}\right)\right)=n_{1}+n_{2}$, then $T_{f}$ has dense 1, 2, 3-type periodic points.

2. If $r\left(\left(n_{1}, n_{2}\right)\right)=n_{1}-n_{2}$, then $T_{f}$ has dense 1, 3, 4-type periodic points.

3. If $r\left(\left(n_{1}, n_{2}\right)\right)=-2 n_{1}+n_{2}$, then $T_{f}$ has dense 1, 2, 4-type periodic points.

4. If $r\left(\left(n_{1}, n_{2}\right)\right)=-5 n_{1}-3 n_{2}$, then $T_{f}$ has dense 2,3 , 4-type periodic points.

We have some corollaries about the conditions of a $\mathbb{Z}^{d}$-action on an infinite metric space $X$ induced by a continuous map $f$ to be $k$-type Devaney chaotic whenever $f$ is Devaney chaotic.

Corollary 2 Let $f$ be a continuous Devaney chaotic on an infinite metric space $Y$ and $T_{f}: \mathbb{Z}^{d} \times Y \rightarrow Y$ be a $\mathbb{Z}^{d}$-action on $Y$ induced by $f$. Suppose $k \in\left\{1,2, \ldots, 2^{d}\right\}$. If there exists $\boldsymbol{m}>^{k} \boldsymbol{O}$ such that $r(\boldsymbol{m})=1$, then $T_{f}$ is $k$-type Devaney chaotic.

Proof Suppose that $(Y, f)$ is Devaney chaotic. Then, $(Y, f)$ is said to be transitive and has dense periodic points. Suppose that there exists $\mathbf{m}>^{k} \mathbf{0}$ such that $r(\mathbf{m})=1$ where $k \in\left\{1,2, \ldots, 2^{d}\right\}$. By Theorem $1, T_{f}$ is $k$-type transitive. Then, for all $s \geq 1$, we must have $\mathbf{n}^{\prime}>^{k} \mathbf{0}$ such that $r\left(\mathbf{n}^{\prime}\right)=s$. By Theorem $4, T_{f}$ has dense $k$-type periodic points. Thus, $T_{f}$ is $k$-type Devaney chaotic.

Corollary 3 Let $f$ be a continuous open Devaney chaotic on an infinite metric space $Y$ and $T_{f}: \mathbb{Z}^{d} \times Y \rightarrow Y$ be $a \mathbb{Z}^{d}$-action on $Y$ induced by $f$. Suppose $k \in\left\{1,2, \ldots, 2^{d}\right\}$. If there exists $m \in \mathbb{N}$ such that for all integers $s \geq m$, there exists $\boldsymbol{n}^{\prime}>^{k} \boldsymbol{0}$ such that $r\left(\boldsymbol{n}^{\prime}\right)=s$ where $\boldsymbol{n}^{\prime} \in \mathbb{Z}^{d}$, then $T_{f}$ is $k$-type Devaney chaotic.

Proof Suppose that $(Y, f)$ is open and Devaney chaotic. Then, $(Y, f)$ is said to be transitive and has dense periodic points. Let $m \in \mathbb{N}$ such that for all integers $s \geq m$, there exists $\mathbf{n}^{\prime}>^{k} \mathbf{0}$ such that $r\left(\mathbf{n}^{\prime}\right)=s$ where $\mathbf{n}^{\prime} \in \mathbb{Z}^{d}$ and $k \in\left\{1,2, \ldots, 2^{d}\right\}$. By Theorem 2, $T_{f}$ is $k$-type transitive. By Theorem $4, T_{f}$ has dense $k$-type periodic points. Thus, $T_{f}$ is $k$-type Devaney chaotic.

\section{Conclusion}

We have introduced a $\mathbb{Z}^{d}$-action, $T_{f}$ on a space $X$ which is induced by a continuous map $f$ on $X$. Counterexamples were given to show that $T_{f}$ does not necessarily inherit all chaos properties from $f$. The study has found some conditions for $T_{f}$ to satisfy 
$k$-type transitive whenever $f$ satisfies some chaos notions such as transitive, totally transitive and topologically mixing. The conditions depend on the homomorphism $r$ which maps from $\mathbb{Z}^{d}$ into $\mathbb{Z}$. Some examples were given to illustrate the findings. We suggest that the future work on $\mathbb{Z}^{d}$-action on $X$ induced by $f$ to be extended by considering other chaos notion such as $k$-type mixing and $k$-type totally transitive. One can proof or disproof if $k$-type mixing on $T_{f}$ can be inherited from the topologically mixing map $f$. Then, one can think of sufficient conditions required for the implication to be satisfied. The work on $k$-type totally transitive can also be done in similar way.

Acknowledgements The authors would like to thank Universiti Kebangsaan Malaysia and Center for Research and Instrumentation (CRIM) for the financial funding through GUP-2019-054.

\section{Declarations}

Conflict of interest The authors declare that they have no conflict of interest.

Open Access This article is licensed under a Creative Commons Attribution 4.0 International License, which permits use, sharing, adaptation, distribution and reproduction in any medium or format, as long as you give appropriate credit to the original author(s) and the source, provide a link to the Creative Commons licence, and indicate if changes were made. The images or other third party material in this article are included in the article's Creative Commons licence, unless indicated otherwise in a credit line to the material. If material is not included in the article's Creative Commons licence and your intended use is not permitted by statutory regulation or exceeds the permitted use, you will need to obtain permission directly from the copyright holder. To view a copy of this licence, visit http://creativecommons.org/licenses/by/4.0/.

\section{References}

1. Banks, J., Brooks, J., Cairns, G., Davis, G., Stacey, P.: On Devaney's definition of chaos. Am. Math. Mon. 99, 332-334 (1992)

2. Barzanouni, A., Divandar, M.S., Shah, E.: On properties of expansive group actions. Acta Math. Vietnam. 44, 923-934 (2019)

3. Kamarudin, N.S., Dzul-Kifli, S.C.: $\mathbb{Z}^{d}$-action induced by shift map on 1-step shift of finite type over two symbols and $k$-type transitive. Math. Stat. 8, 535-541 (2020)

4. Kim, D., Lee, S.: Spectral decomposition of $k$-type nonwandering sets for $\mathbb{Z}^{2}$-actions. Bull. Korean Math. Soc. 51, 387-400 (2014)

5. Oprocha, P.: Chain recurrence in multidimensional time discrete dynamical systems. Discrete Contin. Dyn. Syst. 20, 1039-1056 (2008)

6. Shah, E.: A note on expansive $\mathbb{Z}^{k}$-action and generators. Commun. Korean Math. Soc. 34, 1329-1334 (2019)

7. Shah, S., Das, R.: A note on chaos for $\mathbb{Z}^{d}$-action. Dyn. Contin. Discrete Impuls. Syst. Ser. A Math. Anal. 22, 95-103 (2015)

8. Shah, S., Das, R.: On different types of chaos for $\mathbb{Z}^{d}$-actions. J. Math. Res. 7, 191-197 (2015)

9. Shah, S., Das, R.: On collective sensitivity for $\mathbb{Z}^{d}$-actions. Dyn. Syst. 31, 221-227 (2016)

Publisher's Note Springer Nature remains neutral with regard to jurisdictional claims in published maps and institutional affiliations. 